\title{
Evaluación de la incidencia del síndrome de agotamiento (burnout) en médicos residentes del Centro Médico ABC que trabajan en la primera línea con pacientes COVID-19 con el uso del formulario Maslach Burnout Inventory modificado
}

\section{Evaluation of the incidence of burnout syndrome in residents of the $A B C$ Medical Center working on the frontline with COVID-19 patients with the use of the modified Maslach Burnout Inventory form}

\author{
Lillian Jovana Herrera Parra, ${ }^{*}$ Nora Bernal Ríos, ${ }^{\ddagger}$ Horacio Olivares Mendoza, ${ }^{\ddagger}$ \\ María Fernanda Alarcón Trejo, ${ }^{*}$ Atzin Ruiz Hernández*
}

Citar como: Herrera PLJ, Bernal RN, Olivares MH, Alarcón TMF, Ruiz HA. Evaluación de la incidencia del síndrome de agotamiento (burnout) en médicos residentes del Centro Médico ABC que trabajan en la primera línea con pacientes COVID-19 con el uso del formulario Maslach Burnout Inventory modificado. An Med ABC. 2021; 66 (3): 183-189. https://dx.doi.org/10.35366/101665

\section{RESUMEN}

Introducción: Frente a esta situación crítica los trabajadores de la salud, en especial los médicos residentes en formación en primera línea que participan directamente en el diagnóstico, tratamiento y atención de pacientes con COVID-19, corren el riesgo de desarrollar angustia psicológica y otros síntomas de salud mental. Objetivo: Estimar la incidencia del síndrome de agotamiento (burnout) en médicos residentes del Centro Médico ABC durante la pandemia por COVID-19. Material y métodos: Los datos fueron recolectados por medio de un formulario realizado en Google Forms. Las variables categóricas fueron descritas como frecuencia absoluta y porcentaje. Resultados: Se contestaron 101 formularios. Se puede observar que la incidencia en general del síndrome de agotamiento en los médicos residentes es mayor a tres cuartas partes de la muestra (77\%), una cifra importante del personal

\section{ABSTRACT}

Introduction: Faced with this critical situation, health workers, especially resident doctors in first-line training who are directly involved in the diagnosis, treatment and care of patients with coronavirus-19, are at risk of developing psychological distress and other symptoms of mental health. Objective: To estimate the incidence of burnout syndrome in medical residents of the ABC Medical Center during the coronavirus 19 pandemic. Material and methods: The data was collected through a form made in Google Forms. The categorical variables were described as absolute frequency and percentage. Results: 101 forms were answered. It can be seen that the overall incidence of burnout syndrome in resident physicians is greater than threequarters of the sample (77\%), a significant number of personnel working on the front line during this pandemic. A significant difference was found according to sex, with the female sex
* Residente de Anestesiología.

‡ Anestesiólogo Intensivista.

Centro Médico ABC. México.
Correspondencia: Dra. María Fernanda Alarcón Trejo

E-mail: feralarcontre@gmail.com

Recibido: 04/05/2021. Aceptado: 17/08/2021. 
que trabaja en la primera línea durante esta pandemia. Se encontró una diferencia significativa de acuerdo con el sexo, en el sexo femenino se observó la incidencia más alta, siendo de cerca de $50 \%$, mientras que en el sexo masculino fue de $30 \%$. Conclusión: Todo residente y miembro del personal de la salud es susceptible a este síndrome. Estar conscientes de su existencia e importancia en cuanto al manejo de pacientes y médicos que lo padecen nos debe ayudar a balancear las actividades hospitalarias. Todos los líderes médicos y mentores deben estar conscientes de sus residentes y colegas para asegurar el bienestar de los equipos y de esta manera, contar con un ambiente de trabajo con menos estrés.

Palabras clave: Síndrome burnout, sobrecarga de trabajo, residentes.

Nivel de evidencia: IV

\section{INTRODUCCIÓN}

El coronavirus-19 (COVID-19) ha transformado las vidas de todos los seres humanos en todos los aspectos. Identificado a finales de 2019, la infección por COVID-19 se convirtió rápidamente en una pandemia global en marzo de 2020. Dada la rápida aceleración de transmisión y la falta de preparación para prevenir y tratar esta enfermedad, los efectos negativos del COVID-19 han llegado a cada faceta de la sociedad. ${ }^{1,2}$ La pandemia por COVID-19 es un momento decisivo, los sistemas de salud universales requieren un cambio trascendental para mejorar la preparación ante futuras pandemias, incluida el área de salud mental en los médicos, la cual es un pilar importante para la excelencia en el manejo de pacientes. ${ }^{3,4}$

En áreas hospitalarias se ha observado que hay mayor desgaste personal, laboral y familiar que ha desencadenado mayor estrés; el elevado cansancio emocional y la despersonalización son mayores en personas expuestas a alto nivel de contacto con el sufrimiento y la muerte, teniendo una repercusión negativa en todos los aspectos de la persona en general. ${ }^{3,5}$

Además, hay una inmensidad de problemas identificados como estresantes para los residentes tales como problemas financieros y bajos ingresos, ser evaluados sin la suficiente capacitación y estar bajo presión psicológica y física de sus superiores y pacientes. Así que la depresión y la ansiedad durante la residencia son de esperarse y claramente tienen un impacto en la calidad de la atención de los pacientes. ${ }^{6}$

En un estudio realizado utilizando la base de datos de MEDLINE tomando en cuenta artículos relacionados con el síndrome de agotamiento (burnout) y la residencia médica entre 1990 y 2015 , se encontró una alta prevalencia de dicho síndrome en estudian- having the highest incidence of this being close to 50\%, while in the male sex it was $30 \%$. Conclusion: Each resident and any member of the health personnel are susceptible to it, being aware of its existence and importance in terms of the management of patients in doctors who suffer from it should help us to balance hospital activities. All medical leaders and mentors must be mindful of their residents and colleagues to ensure the wellbeing of the teams for a less stressful work environment.

Keywords: Burnout syndrome, work overload, residents.

Level of evidence: $I V$

tes y médicos residentes con incidencias de $40-60 \%$, a pesar de que se cree que esta incidencia ha aumentado con el tiempo, es difícil de determinar debido a la falta de estudios realizados recientemente. ${ }^{7}$

Frente a esta situación crítica los trabajadores de la salud, en especial los médicos residentes en formación en primera línea que participan directamente en el diagnóstico, tratamiento y atención de pacientes con COVID-19, corren el riesgo de desarrollar angustia psicológica y otros síntomas de salud mental. El número cada vez mayor de casos confirmados y sospechosos, la carga de trabajo abrumadora, el agotamiento de los equipos de protección personal, la cobertura generalizada de los medios, la falta de medicamentos específicos y la sensación de recibir apoyo inadecuado pueden contribuir a la carga mental de todos los trabajadores de la salud, a esto hay que sumar que el médico residente de México, el cual, independientemente de su especialidad, debido a la situación previamente mencionada, se vio obligado a hacer un cambio drástico en el programa académico y actividades hospitalarias esperadas durante su año.

Estudios anteriores informaron reacciones psicológicas adversas al brote de SARS de 2003 entre los trabajadores de la salud. Los estudios mostraron que dichos trabajadores temían el contagio y la infección a sus familiares, amigos y colegas, sentían incertidumbre y estigmatización, mostraron renuencia a trabajar o aceptaron la resignación, y manifestaron experimentar altos niveles de síntomas de estrés, ansiedad y depresión, lo que podría tener implicaciones psicológicas a largo plazo. ${ }^{8}$

En consecuencia, la literatura con la que contamos sobre estrés en el personal médico y hospitalario en general se basa en el uso de encuestas, cuestionarios y entrevistas, las cuales nos ayudan a determi- 
nar las percepciones de los residentes, su formación y ambiente de trabajo; sin embargo, estos reportes están repletos de los problemas típicos de las medidas de autoinformes. ${ }^{6}$

Este síndrome ha sido ampliamente estudiado en el área de la salud y se le ha asociado a diversas consecuencias profesionales y personales. Se han llevado a cabo numerosos estudios para estimar los porcentajes de agotamiento (burnout) en profesionales de la salud, reportando prevalencias de 25 a $75 \%$. $^{9}$

El síndrome de agotamiento (burnout) fue descrito por Freudenberger, ${ }^{10}$ pero fue Maslach quien en 1976 diseñó un cuestionario para diagnosticarlo y lo organizó en tres dimensiones: el agotamiento emocional (caracterizado por la pérdida progresiva de energía y recursos personales de adaptación); la despersonalización (manifestada por un cambio negativo de actitudes que lleva una respuesta fría e impersonal y falta de sentimientos e insensibilidad hacia los pacientes), y la realización profesional (que describe sentimientos de competencia y eficacia en el trabajo). ${ }^{11}$ Los síntomas del agotamiento (burnout) varían de un individuo a otro, pero también varían dependiendo de la etapa del síndrome.

Objetivo: estimar la incidencia del síndrome de agotamiento (burnout) en médicos residentes del Centro Médico ABC durante la pandemia por COVID-19.

\section{MATERIAL Y MÉTODOS}

Estudio analítico, prospectivo, transversal, no comparativo. El objetivo principal fue calcular la incidencia de síndrome por agotamiento (burnout) en los médicos residentes del Centro Médico $\mathrm{ABC}$ que trabajan en áreas COVID y no COVID durante el periodo de abril-julio de 2020. Asimismo, estratificar estos resultados para comparar las poblaciones en las que la incidencia de este síndrome es mayor.

Posterior a la aceptación del protocolo por el comité de ética, se invitó a los médicos residentes del Centro Médico ABC a contestar el cuestionario Maslach Burnout Inventory modificado. Los datos fueron recolectados por medio de un formulario realizado en Goo- gle Forms. Las variables categóricas serán descritas como frecuencia absoluta y porcentaje.

Para realizar el diagnóstico del síndrome de agotamiento, como se mencionó previamente la herramienta aprobada y utilizada es el cuestionario generado por Maslach, que está constituido por 22 ítems en forma de afirmaciones sobre los sentimientos y actitudes del profesional en su trabajo y en este caso, hacia los pacientes, su función es medir el desgaste profesional. Este formulario pretende medir la frecuencia y la intensidad con la que se padece el síndrome de agotamiento (burnout).

Para hacer el diagnóstico del síndrome de agotamiento (burnout) es necesario obtener cierta puntuación en cualquiera de las tres subescalas evaluadas, o puede obtenerse en más de una. A continuación se explica de manera detallada la manera de evaluación y calificación del formulario (Tablas 1 y 2).

A raíz de todos los estudios realizados podemos concluir que la pandemia nos ha llevado a numerosos casos de estrés, ansiedad, depresión, síndrome de agotamiento y exacerbaciones de enfermedades mentales subyacentes, no sólo en el personal de salud y los médicos residentes, sino en toda la población. ${ }^{12}$

Criterios de inclusión: médicos residentes del Centro Médico ABC durante el periodo de marzo de 2020 a febrero de 2021.

Criterios de exclusión: los médicos que se negaron a contestar el formulario.

Tamaño de la muestra: este estudio epidemiológico no tiene definido ningún desenlace y debido a que no se tiene un estudio previo para estimar el tamaño de la muestra, se incluyeron todos los médicos residentes que contestaron el formulario dentro del periodo previamente mencionado.

Todas las variables continuas se expresaron como media \pm desviación estándar (DE). Las medidas categóricas se expresaron como número (\%). La prueba de normalidad se realizó con Shapiro-Wilk. Utilizamos pruebas paramétricas o no paramétricas, según fuera apropiado para comparar las diferencias en las características, además de realizar razón de momios con intervalos de confianza (IC) de 95\%. Toda la esta-

Tabla 1: Cálculo de puntuaciones para síndrome de agotamiento.

\begin{tabular}{ccc}
\hline Aspecto evaluado & Preguntas evaluadas & Indicios de burnout \\
\hline Agotamiento/cansancio emocional & $1,2,3,6,8,13,14,16,20$ & Más de 26 \\
Despersonalización & $5,10,11,15,22$ & Más de 9 \\
Realización personal & $4,7,9,12,17,18,19,21$ & Menos de 34 \\
\hline
\end{tabular}


Tabla 2: Valores de referencia para clasificación de severidad de síndrome de agotamiento (burnout).

\begin{tabular}{llcl}
\hline Aspecto por evaluar & Bajo & Medio & Alto \\
\hline Agotamiento/CE & $0-18$ & $19-26$ & $27-54$ \\
Despersonalización & $0-5$ & $6-9$ & $10-30$ \\
Realización personal & $0-33$ & $34-39$ & $40-56$ \\
\hline CE = cansancio emocional. & & &
\end{tabular}

dística se realizó con el software Prism (versión 6.0). Se consideró significación estadística si $\mathrm{p} \leq 0.05$.

\section{RESULTADOS}

Se recolectaron los resultados de los formularios Maslach Burnout Inventory modificado en la plataforma de Google Forms llenados por los médicos residentes del Centro Médico ABC en el periodo mayojulio de 2020 .

Se contestaron 101 formularios durante el periodo establecido, cumpliendo con los criterios de inclusión. De los 101 participantes, 54 (53.6\%) fueron del sexo femenino y 47 (46.5\%) del sexo masculino, con una media de edad de 27.7 años con desviación estándar de \pm 2.05 .

En la Tabla 3 se muestran los datos sociodemográficos de la población del estudio y su clasificación de acuerdo a las variables a comparar como sexo, edad, especialidad, campus y áreas de trabajo.

Se divide la población de acuerdo al tipo de síndrome de agotamiento (burnout), se clasificaron siete tipos, observándose la mayor proporción de la población en el tipo que abarca todas las dimensiones del síndrome (Tabla 4).

En la Tabla 5 se muestran las especialidades médicas, se hizo una división entre la población con síndrome de agotamiento (burnout) y la que no cumple con los criterios para su diagnóstico.

En la Tabla 6 se divide la población del estudio por áreas de trabajo y se correlacionó la incidencia de síndrome por agotamiento (burnout), encontrando que ésta fue mayor de $80 \%$ en manejo de pacientes COVID-19.

\section{DISCUSIÓN}

Actualmente las residencias médicas se han visto afectadas por los cambios y la incertidumbre, sobre todo a nivel académico, que esta pandemia representa. El estrés en todo el personal médico ha aumentado. En tiempos de incertidumbre, la ciencia es una de las pocas cosas que nos puede ayudar a encontrar claridad en medio del caos. ${ }^{2}$

En cuanto a los resultados del estudio, lo que se puede observar es que la incidencia en general del

Tabla 3: Datos sociodemográficos.

\begin{tabular}{|c|c|}
\hline & $n(\%)$ \\
\hline \multicolumn{2}{|l|}{ Sexo } \\
\hline Femenino & $54(53.6)$ \\
\hline Masculino & $47(46.5)$ \\
\hline Edad (años) & $27.7 \pm 2.05^{*}$ \\
\hline \multicolumn{2}{|l|}{ Burnout general } \\
\hline Sí & $77(75.5)$ \\
\hline No & $24(24.5)$ \\
\hline \multicolumn{2}{|l|}{ Especialidad } \\
\hline Anestesiología & $14(13.8)$ \\
\hline Cardiología & $2(1.9)$ \\
\hline Cirugía General & $4(3.9)$ \\
\hline Geriatría & $8(7.9)$ \\
\hline Ginecología & $10(9.9)$ \\
\hline Imagen Diagnóstica & $10(9.9)$ \\
\hline Medicina Crítica & $5(4.9)$ \\
\hline Urgencias & $1(0.9)$ \\
\hline Medicina Interna & $31(30.7)$ \\
\hline Oncología Médica & $2(1.9)$ \\
\hline Ortopedia y Traumatología & $14(13.8)$ \\
\hline \multicolumn{2}{|l|}{ Año de especialidad } \\
\hline Primero & $19(18.8)$ \\
\hline Segundo & $37(36.6)$ \\
\hline Tercero & $26(25.7)$ \\
\hline Cuarto & $13(12.8)$ \\
\hline Quinto & $2(1.9)$ \\
\hline Sexto & $2(1.9)$ \\
\hline \multicolumn{2}{|l|}{ Horas de trabajo } \\
\hline$\leq 12$ & $22(21.8)$ \\
\hline $12-24$ & $36(35.6)$ \\
\hline $24-36$ & $43(42.5)$ \\
\hline \multicolumn{2}{|l|}{ Horas de descanso } \\
\hline$\leq 12$ & $1(0.9)$ \\
\hline $12-24$ & $13(12.8)$ \\
\hline $24-36$ & $43(42.5)$ \\
\hline $36-48$ & $34(33.6)$ \\
\hline$>48$ & $10(9.9)$ \\
\hline \multicolumn{2}{|l|}{ Área de trabajo } \\
\hline Triaje respiratorio & $12(11.8)$ \\
\hline Urgencias Respiratorias & $2(1.9)$ \\
\hline Urgencias & $9(8.9)$ \\
\hline Hospitalización & $29(28.7)$ \\
\hline Terapia Intensiva & $26(25.7)$ \\
\hline Quirófano & $4(3.9)$ \\
\hline Otro & $19(18.8)$ \\
\hline \multicolumn{2}{|l|}{ Campus } \\
\hline Observatorio & $55(54.4)$ \\
\hline Santa Fe & $46(45.5)$ \\
\hline
\end{tabular}


Tabla 4: Tipos de síndrome de agotamiento (burnout).

\begin{tabular}{crc}
\hline & $n$ & $\%$ \\
\hline CE & 12 & 15.58441558 \\
CE + D & 6 & 7.792207792 \\
CE + D + RP & 31 & 40.25974026 \\
CE + RP & 20 & 25.97402597 \\
D & 2 & 2.597402597 \\
D + RP & 1 & 1.298701299 \\
RP & 5 & 6.493506494 \\
\hline CE = cansancio emocional, D = despersonalización, RP = realización personal.
\end{tabular}

síndrome de agotamiento en los médicos residentes del Centro Médico ABC durante la pandemia es mayor de tres cuartas partes de la muestra (77\%), una cifra importante del personal que trabaja en la primera línea durante esta pandemia. A diferencia de estudios realizados en tiempos prepandemia COVID-19, la incidencia entre médicos residentes con síndrome de agotamiento (burnout) ha aumentado $25-30 \% .^{7}$

Se encontró una diferencia significativa en la presentación de acuerdo con el sexo, en el sexo femenino hay una incidencia más alta, siendo cerca de $50 \%$, mientras que en el sexo masculino fue de $30 \%$. Estos resultados coincidieron con el estudio realizado por la Universidad de Cauca, Colombia en 2018, donde se concluyó de acuerdo con sus resultados que la prevalencia para este síndrome y sexo fue de 0.9 , en el sexo femenino fue mayor en relación con el masculino. ${ }^{7}$

Todas las especialidades incluidas en el estudio mostraron porcentajes mayores de 50\% del síndrome de agotamiento (burnout). Dentro de éstas, ginecología y obstetricia mostró el porcentaje más bajo $50 \%$ y las especialidades de cardiología, medicina crítica, medicina de urgencias y oncología tuvieron un porcentaje de $100 \%$ de residentes con síndrome de agotamiento (burnout). En cuanto a los porcentajes encontrados de síndrome de agotamiento (burnout) en las diferentes especialidades, podemos observar claramente mayor incidencia en las especialidades que trabajaron en la primera línea durante esta pandemia, a diferencia de los estudios realizados previos a la pandemia donde predomina este síndrome en especialidades quirúrgicas con jornadas más largas dentro de quirófano. ${ }^{13,14}$

De los diferentes tipos de presentación del síndrome de agotamiento (burnout), se observó $40 \%$ de los residentes con diagnóstico de síndrome de agotamiento (burnout), éste se compuso de tres aspectos (cansancio emocional, despersonalización y realización personal), mientras que los tipos menos comunes de síndrome de agotamiento (burnout) fue el compuesto sólo por despersonalización con $2 \%$ y el compuesto por los aspectos de despersonalización y realización personal $1 \%$.

De acuerdo con el área de trabajo de los médicos residentes, se observó mayor incidencia en áreas que manejan pacientes COVID-19 que en áreas de bajo riesgo, siendo el área de terapia intensiva la que tuvo la mayor incidencia con $92 \%$, seguida de hospitalización en centro COVID-19 con $89 \%$ y las áreas con menor incidencia fueron áreas como rayos X con un porcentaje de incidencia de $47 \%$. Como se mencionó previamente, éste fue uno de los resultados con más importancia dentro del presente estudio debido a su diferencia significativa, la cual mostró que el residente que trabaja en estas áreas tiene 1.5 veces más el riesgo de padecer síndrome de agotamiento (burnout) que si trabajara en otras áreas libres de pacientes COVID-19. Este estudio se suma a la lista de los que se han realizado durante la pandemia y demuestra, junto con ellos, que la prevalencia del síndrome de agotamiento (burnout) en médicos residentes es alta, comprobando que la amenaza que representa la pandemia COVID-19 es un estresante importante para el personal médico. ${ }^{12,15}$

Como limitaciones de nuestro estudio, puede decirse que éste fue unicéntrico y no participó $100 \%$ de la población de médicos residentes dentro de la sede, por lo que los resultados pueden variar mucho de acuerdo al porcentaje que participó. Otra limitación fue la temporalidad del estudio, ya que se realizó sólo a comienzos de la pandemia, sólo a dos o cinco meses de haber comenzado, por lo que

Tabla 5: Incidencia del síndrome de agotamiento por servicio médico.

\begin{tabular}{lrrc}
\hline Especialidad & Sí & No & Promedio \\
\hline Anestesiología & 12 & 14 & 85.71428571 \\
Cardiología & 2 & 2 & 100 \\
Cirugía General & 3 & 4 & 75 \\
Geriatría & 5 & 8 & 62.5 \\
Ginecología y Obstetricia & 5 & 10 & 50 \\
Imagen Diagnóstica y Terapéutica & 6 & 10 & 60 \\
Medicina Crítica & 5 & 5 & 100 \\
Medicina de Urgencias & 1 & 1 & 100 \\
Medicina Interna & 27 & 31 & 87.09677419 \\
Oncología & 2 & 2 & 100 \\
Trauma & 9 & 14 & 64.28571429 \\
\hline
\end{tabular}


Tabla 6: Incidencia del síndrome de agotamiento (burnout) por área de trabajo.

\begin{tabular}{lrrcr}
\hline & Sí & No & Promedio & Total \\
\hline Terapia Respiratoria & 5 & 7 & 41.66666667 & 12 \\
Unidades Respiratorias & 2 & 0 & 100 & 2 \\
Urgencias & 7 & 2 & 77.77777778 & 9 \\
Hospitalización & 26 & 3 & 89.65517241 & 29 \\
Unidad de Terapia Intensiva & 24 & 2 & 92.30769231 & 26 \\
Quirófano & 3 & 1 & 75 & 4 \\
Otros & 9 & 10 & 47.36842105 & 19 \\
\hline
\end{tabular}

sería conveniente realizar un comparativo o un seguimiento posterior.

Como fortalezas, consideramos que este tipo de estudio a nivel de nuestro país, el cual como se mencionó previamente cuenta con los horarios más extensos para sus médicos residentes, no se había realizado en instituciones privadas en el pasado, por lo que se puede usar como punto de referencia y comparación para estudios en el futuro. Otra fortaleza son los resultados que indican que la incidencia del síndrome de agotamiento (burnout) es alarmante, lo que puede usarse como plataforma para difundir información y generar interés para su manejo en el futuro.

\section{CONCLUSIONES}

La existencia de protocolos para cada tipo de situación, el entrenamiento del personal en cuanto a medidas de protección y todas las medidas para asegurar un adecuado nivel de confianza y control obviamente disminuye el estrés.

También es importante la unión entre todo el personal de la salud para encontrar maneras de disminuir el estrés y contar con un apoyo psicológico. La energía en todas las áreas de trabajo durante la pandemia debe ser positiva. Es importante que haya periodos de descanso y relajación para prevenir este síndrome, aunque esto a menudo sea lejos de nuestros seres queridos y familias.

Debemos tener en cuenta que el síndrome de agotamiento (burnout) es real y tiene muchas maneras de manifestarse. Todo residente y miembro del personal de la salud es susceptible de sufrirlo, estar conscientes de su existencia e importancia en cuanto al manejo de pacientes y médicos que lo padecen nos debe ayudar a balancear las actividades hospitalarias. Todos los líderes médicos y mentores deben estar conscientes de sus residentes y colegas para asegurar el bienestar de los equipos y de esta manera contar con un ambiente de trabajo con menos estrés.

En el contexto de COVID-19 la mejor manera de combatir el síndrome de agotamiento (burnout) es encontrar la forma de entrenar al personal para que se sienta lo más seguro posible no sólo respecto al equipo de protección personal, sino al entrenamiento mental sobre técnicas para disminuir el estrés y promover la salud mental así como desarrollar actividades hospitalarias con retroalimentación positiva en todo momento.

Este estudio es sólo el comienzo, se busca alentar a los médicos residentes de nuestro país a utilizarlo como base para estudios sobre el síndrome de agotamiento en nuestra población y de esta manera generar conciencia y mejorar el sistema de residencias médicas.

\section{REFERENCIAS}

1. Jackson SH. The role of stress in anaesthetists' health and well-being. Acta Anaesthesiol Scand. 1999; 43 (6): 583-602.

2. Horesh D, Brown AD. Traumatic stress in the age of COVID-19: A call to close critical gaps and adapt to new realities. Psychol Trauma. 2020; 12 (4): 331-335.

3. Gutiérrez AGA, Celis LMA, Moreno JS, Farias SF, Suárez CJJ. Síndrome de burnout. Arch Neurocien. 2006; 11 (4): 305-309.

4. Zaka A, Shamloo SE, Fiorente P, Tafuri A. COVID-19 pandemic as a watershed moment: A call for systematic psychological health care for frontline medical staff. J Health Psychol. 2020; 25 (7): 883-887.

5. Carvajal Carrasco C. El síndrome de Burnout, una nueva realidad en la educación chilena. Comunicaciones en Humanidades. 2017; 1 (2): 71-81.

6. Butterfield PS. The stress of residency. A review of the literature. Arch Intern Med. 1988; 148 (6): 1428-1435.

7. Jácome SJ, Villaquiran-Hurtado A, García CP, Duque IL. Prevalencia del síndrome de Burnout en residentes de especialidades médicas. Rev Cuid. 2019; 10 (1): e543.

8. Lai J, Ma S, Wang Y, Cai Z, Hu J, Wei N et al. Factors associated with mental health outcomes among health care workers exposed to coronavirus disease 2019. JAMA Netw Open. 2020; 3 (3): e203976. 
An Med ABC. 2021; 66 (3): 183-189

9. Doulougeri K, Georganta K, Montgomery A. "Diagnosing" burnout among healthcare professionals: can we find consensus? Cogent Med. 2016; 3 (1): 1-10.

10. Freudenberger HJ. Staff burnout. J Soc Issues. 1974; 30: 159-165.

11. Maslach C, Schaufeli WB, Leiter MP. Job burnout. Annu Rev Psychol. 2001; 52: 397-422.

12. Dimitriu MCT, Pantea-Stoian A, Smaranda AC, Nica AA, Carap AC, Constantin VD et al. Burnout syndrome in Romanian medical residents in time of the COVID-19 pandemic. Med Hypotheses. 2020; 144: 109972.
13. Dyrbye L, Shanafelt T. A narrative review on burnout experienced by medical students and residents. Med Educ. 2016; 50 (1): 132-149.

14. López-Morales A, González-Velázquez F, Morales-Guzmán MI, Espinoza-Martínez CE. Síndrome de burnout en residentes con jornadas laborales prolongadas. Rev Med Inst Mex Seguro Soc. 2007; 45 (3): 233-242.

15. Ebrahimi S, Kargar Z. Occupational stress among medical residents in educational hospitals. Ann Occup Environ Med. 2018; 30: 51. 